\title{
Comparison of 3DVH Software with Two-dimensional Array Systems on Pretreatment Verification for Volumetric-modulated Arc Therapy
}

\author{
Ji Hoon Park', Tae Gyu Kim¹, Soon Ki Min² and Byung Do Park* \\ ${ }^{1}$ Department of Radiation Oncology, Samsung Changwon Hospital, Sungkyunkwan University School of Medicine, Changwon, Republic of Korea \\ ${ }^{2}$ Proton Therapy Center, National Cancer Center, Goyang, Republic of Korea
}

\begin{abstract}
Objectives: The purpose of this study is to investigate the comparison of 3DVH software and two-dimensional array systems (MatriXX, ArcCHECK, and portal dosimetry system) on pretreatment verification for volumetricmodulated arc therapy.

Methods: Dosimetric measurements were performed using the verification for 20 treatment plans. Measured dosimetric differences were evaluated by gamma pass rate and percentage dose difference. Dose-volume histograms (DVHs) calculated by the treatment planning system were also compared with those predicted by the 3DVH software.

Results: The mean gamma pass rates were more than $95 \%$ for the $3 \% / 3 \mathrm{~mm}$ criterion, except for 3D evaluation using the 3DVH (3DVH (3D)) software in prostate cancer cases. In the cases of head-and-neck (HN) cancer, the mean gamma pass rates by ArcCHECK and 3DVH 2D evaluation (3DVH (2D)) were estimated to be lower than those of MatriXX, EPID, and 3DVH (3D) for the $2 \% / 2 \mathrm{~mm}$ and $1 \% / 1 \mathrm{~mm}$ criteria. The percentage dose differences were within $4 \%$ for all structures, and correlated with the mean gamma pass rate for the planning target volume (PTV) and the $D_{\text {mean }}$ of the spinal cord $(p<0.05)$. On the other hand, the mean gamma pass rates of prostate cases presented similar results for all criteria. The percentage dose differences for structure volumes in the cases of prostate cancer (from $2.76 \%$ to $12.58 \%$ ) were larger than those in the cases of $\mathrm{HN}$ cancer, and there was no statistical significance except for the $D_{\text {max }}$ of the bladder.

Conclusion: Judging from our results, the three dosimetric devices showed similar results for pretreatment verification and portal dosimetry can be replaced as the verification system. However, the use of 3DVH software remains to be a matter for further discussion.
\end{abstract}

Keywords: VMAT; 3DVH; ArcCHECK; MatriXX; Portal dosimetry

\section{Introduction}

Three-dimensional conformal radiotherapy (3DCRT) was made possible by the development of computed tomography (CT). According to this procedure, the 3DCRT plan is created manually with beam parameters such as the number of beams, the direction of the beams, the shape of the beams, the angle of the wedge, the weight of the beams, etc. [1]. However, for radiation therapy using this technique, it is difficult to adjust the dose for normal organs close to the target volume. On the other hand, intensity-modulated radiation therapy (IMRT) is an advanced type of 3DCRT. The IMRT technique is optimized for the protection of the healthy tissue and the improvement of dose coverage for complex target volumes. This technique has supplied results superior to those of 3DCRT in terms of target coverage, homogeneity, and conformity for various lesions [2-5]. Meanwhile, the volumetric-modulated arc therapy (VMAT) technique, which involves gantry rotation, can reduce the treatment time and intrafractional error because of mean monitor unit (MU) less than that of the IMRT technique [6]. The treatment plans using the IMRT or VMAT techniques are automatically established through an optimization process. Therefore, these techniques are able to control the dose constraint of the target volume and normal tissue in the optimization process and this process improves the target coverage and dose distribution for normal tissue. The fluence of the treatment plan using IMRT or VMAT depends on the accuracy of the position of the gantry, collimator, and multileaf collimator (MLC) and reproducibility of their movement. Therefore, it is recommended that the IMRT and VMAT plans be verified before treatment in some studies $[7,8]$. The goal of pretreatment verification is to confirm the state of treatment machine and the accuracy of optimized treatment plan.
In the past, the verification of radiation treatment plan was performed by using an ionizing chamber and a film dosimetry [9]. However, recent two-dimensional array systems such as MatriXX (IBA Dosimetry Schwarzenbruck, Germany) and ArcCHECK (Sun Nuclear Corporation, Melbourne, FL, USA) dosimetry systems were produced for pretreatment quality assurance (QA). Portal dosimetry system that uses an electronic portal imaging device (EPID) is also very simple method for obtaining dose information of the verification plan. However, these devices simply measure and evaluate the $2 \mathrm{D}$ dose distribution through gamma analysis; but the controversial issue at hand is to verify the dose for the target volume and some organ at risk (OAR).

Nelms et al. proposed the evaluation of the dose-volume histogram (DVH)-based metric for patient-specific QA [10]. The 3DVH software (Sun Nuclear Corporation) was developed to evaluate the

\footnotetext{
*Corresponding author: Byung Do Park, Department of Radiation Oncology, Samsung Changwon Hospital, Sungkyunkwan University School of Medicine, Changwon, Republic of Korea, Tel: +82-55-290-1322; Fax: +82-55-290-1327; E-mail: byungdo.park@gmail.com

Received February 19, 2016; Accepted March 25, 2016; Published March 29, 2016

Citation: Park JH, Kim TG, Min SK, Park BD (2016) Comparison of 3DVH Software with Two-dimensional Array Systems on Pretreatment Verification for Volumetricmodulated Arc Therapy. J Nucl Med Radiat Ther 7: 284. doi:10.4172/21559619.1000284

Copyright: $\odot 2016$ Park JH, et al. This is an open-access article distributed under the terms of the Creative Commons Attribution License, which permits unrestricted use, distribution, and reproduction in any medium, provided the original author and source are credited.
} 
Citation: Park JH, Kim TG, Min SK, Park BD (2016) Comparison of 3DVH Software with Two-dimensional Array Systems on Pretreatment Verification for Volumetric-modulated Arc Therapy. J Nucl Med Radiat Ther 7: 284. doi:10.4172/2155-9619.1000284

Page 2 of 6

dose distribution for each organ volume through comparison of the DVHs. Keeling et al. subsequently reported the correlation between DVH-based QA metrics and the gamma passing rate using the $3 \mathrm{DVH}$ software and the MapCHECK2 detector [11]. The 3DVH software was evaluated in terms of the correlation between the percentage dose difference (\%DD) and the gamma pass rate by Infusino et al., and the evaluation of the accuracy of this software was performed by Olch $[12,13]$. They established the grade of correlation using the correlation coefficient, and their results showed weak-to-moderate correlation for all structures. Song et al. then reported that the 3D gamma pass rate using the 3DVH software is influenced by the target size [14]. However the $3 \mathrm{DVH}$ software should be more investigated for various dosimetric and clinical benefits, and this advanced software should be evaluated for various lesions with heterogeneous structures. The purpose of this study is to investigate the comparison of $3 \mathrm{DVH}$ software and twodimensional array systems on pretreatment verification for cases of head-and-neck (HN) and prostate cancer.

\section{Method}

Pretreatment verification was carried out for 20 VMAT plans, whose treatment regions corresponded to $\mathrm{HN}$ and prostate cancer. Beam delivery was performed in a Varian linear accelerator with 6-MV and 10-MV. The dose distributions of all VMAT plans were obtained by MatriXX 2D ion chamber array, ArcCHECK 2D diode array and EPID.

Firstly, verification plans were created using the MatriXX 2D ion chamber array and slab phantom in Eclipse v11 (Varian Medical Systems, Palo Alto, CA, USA). The MatriXX detector consists of 1020 ionization chambers distributed in throughout active area of 24 $\times 24 \mathrm{~cm}^{2}$. Each chamber has a diameter of $4.5 \mathrm{~mm}$ and a height of 5 $\mathrm{mm}$. The center-to-center distance between two adjacent detectors is $7.62 \mathrm{~mm}$. The manufacturer recommends a warm-up time of more than $15 \mathrm{~min}$. Furthermore, $1000 \mathrm{MU}$ of pre-irradiation is needed before acquiring the dose distribution of the verification plan. The correction factor (kuser) for the energy should be measured because the MatriXX detector depends on the selected treatment machine, energy type, pressure, and temperature. The kuser factor is obtained at the maximum depth and $100 \mathrm{~cm}$ source-to-surface distance for used energy. The MatriXX detector was positioned within solid water slabs of backup and build-up material to acquire the dose distribution. The measured dose distributions for each verification plan were evaluated using gamma analysis with OmniPro-I'mRT software (IBA Dosimetry Schwarzenbruck, Germany).

Secondly, verification of VMAT plans was performed with the EPID (aS1000) that was installed on the linear accelerator with arrays of light-sensitive amorphous silicon photodiodes arranged in an active detector area of $40 \times 30 \mathrm{~cm}^{2}(1024 \times 768$ pixels $)$. Therefore, the dose distribution was acquired without the use of another device. Images were acquired using a frame-averaging method with a fast framegrabbing rate of 30 frames per second. The dose normalization was performed in calibration unit (CU), with $1 \mathrm{CU}$ being equal to $1 \mathrm{~Gy}$ (i.e., $1 \mathrm{CU}=1 \mathrm{~Gy}$ ), before obtaining the dose distribution. Last but not least, verification plans were generated by the ArcCHECK diode array, a cylindrical array that consists of 1386 diode detectors. The measured dose distribution was analyzed by using SNC patient application (Sun Nuclear Corporation, Melbourne, FL, USA) and 3DVH software.

$3 \mathrm{DVH}$ software evaluated the dosimetric difference with the ArcCHECK planned dose perturbation (ACPDP) calculator (Figure 1). The ACPDP algorithm is a novel method for estimating the dose distribution and DVHs for a contoured structure [15].
The software requires the patient plan DICOM files (RT Plan, RT Dose, RT Structure, and planning CT images), an ArcCHECK movie lite $\left({ }^{*}\right.$.acml) file, and a planned file with ArcCHECK to compare the dose difference between the ArcCHECk measurement and the treatment planning system calculation. The dose predicted by the ACPDP was calculated from the patient plan DICOM files and the phantom plan DICOM files. These dose distributions could then be compared in terms of the DVH of each structure with the measured dose distribution. Furthermore, the gamma pass rate was used to quantify the agreement between the calculations and measurements (Figure 2).

AAPM TG 119 and many studies have investigated acceptance levels of patient QA, and they have suggested a 3\% dose difference and $3 \mathrm{~mm}$ distance-to-agreement criterion [16]. In this paper, measured dose distributions were evaluated with $1 \% / 1 \mathrm{~mm}, 2 \% / 2 \mathrm{~mm}$, and $3 \% / 3$ $\mathrm{mm}$ criteria. The percentage dose difference (\%DD) was calculated for the planning target volume (PTV) and normal organs with 3DVH software. For each PTV, the mean dose $\left(\mathrm{D}_{\text {mean }}\right)$, maximum dose $\left(\mathrm{D}_{\max }\right)$, as well as the dose received by $2 \%, 95 \%$, and $98 \%$ of the volume (D2\%, D95\%, and D98\%)-which were predicted by ACPDP-were compared with those of the treatment planning system. Normal organs were evaluated according to the values of their $\mathrm{D}_{\text {mean }}$ and $\mathrm{D}_{\max }$. The $\% \mathrm{DD}$ is defined as follows:

$$
\% \mathrm{DD}=(\mathrm{D} 3 \mathrm{DVH}-\mathrm{DTPS}) / \mathrm{DTPS} \times 100 .
$$

$\mathrm{D}_{3 \mathrm{DVH}}$ represents the dose by $3 \mathrm{DVH}$, whereas $\mathrm{D}_{\text {TPS }}$ represents the dose calculated by Eclipse v11. The correlations between the gamma pass rate $(3 \% / 3 \mathrm{~mm}$ criterion) and \%DD were examined with SPSS v18 (SPSS Inc., Chicago IL).

\section{Results}

Obtained dose distributions were evaluated with gamma index, and $3 \mathrm{DVH}$ software provides the $2 \mathrm{D}$ and $3 \mathrm{D}$ gamma index $(3 \mathrm{DVH}$ (2D) and $3 \mathrm{DVH}(3 \mathrm{D})$ ). Table 1 shows the mean gamma pass rates and standard deviations. The mean gamma pass rates were above $95 \%$ for the $3 \% / 3 \mathrm{~mm}$ criterion, except for the gamma value of $3 \mathrm{DVH}(3 \mathrm{D})$. In the HN cancer cases, The mean gamma pass rates of ArcCHECK and $3 \mathrm{DVH}(2 \mathrm{D})$ were estimated to be lower than those of MatriXX, portal

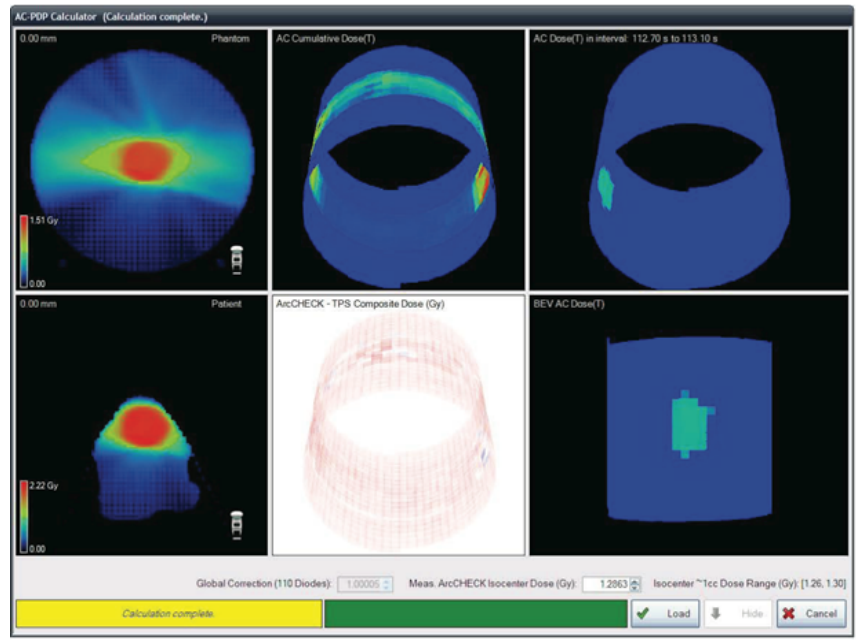

Figure 1: ArcCHECK planned dose perturbation (ACPDP) calculation in 3DVH software (Sun nuclear). 

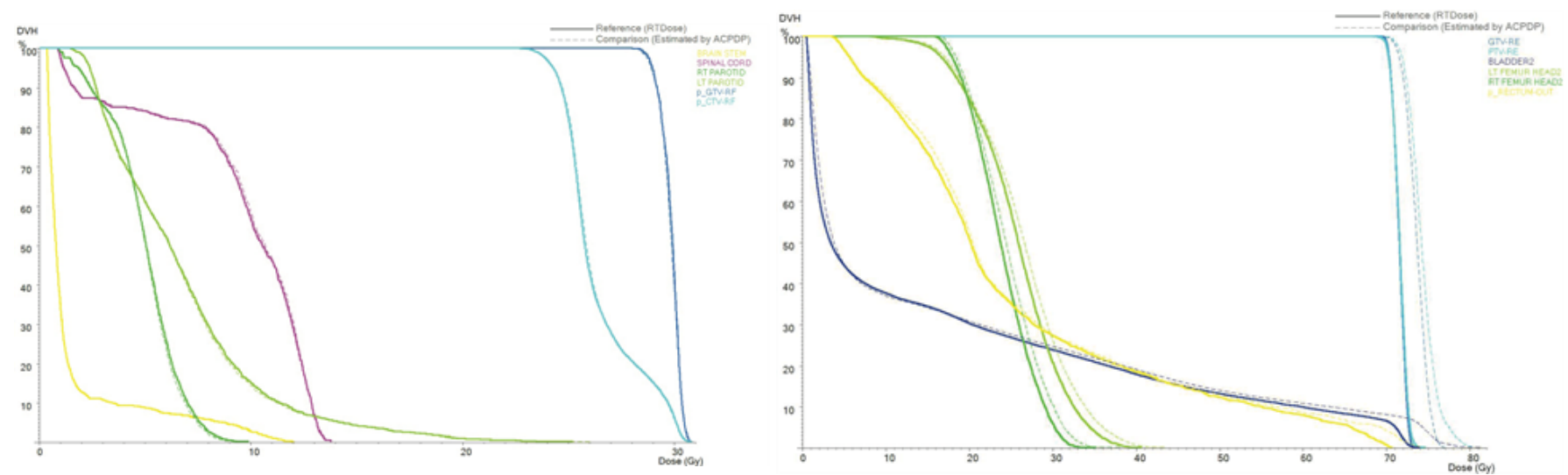

Figure 2: An example of a dose-volume histogram (DVH) for the target and structures. Dotted lines represent DVHs using the ArcCHECK planned dose perturbation (ACPDP) algorithm, and solid lines represent DVHs calculated in eclipse v11. Left: Head-and-Neck (HN) Cancer Cases; Right: Prostate Cancer Cases.

dosimetry, and $3 \mathrm{DVH}(3 \mathrm{D})$ with $2 \% / 2 \mathrm{~mm}$ and $1 \% / 1 \mathrm{~mm}$ criteria. Whereas, the mean gamma values presented similar tendencies in each criterion for the cases of prostate cancer.

Table 2 shows the \%DD for both ACPDP and eclipse v11, as well as the correlation between the $\% \mathrm{DD}$ and the mean gamma pass rate as evaluated by $3 \mathrm{DVH}(3 \mathrm{D})$. We also analyzed the dose difference and correlation for target and normal organs of $\mathrm{HN}$ and prostate cancer cases. In $\mathrm{HN}$ cases, the percentage dose difference was less than $2 \%$ for target volume, and $4 \%$ for normal organs. The percentage dose difference was correlated with the gamma pass rate for $\mathrm{D}_{\text {mean }}, \mathrm{D}_{\max }$, $\mathrm{D} 2 \%, \mathrm{D} 95 \%$, and $\mathrm{D} 98 \%$ of PTV and $\mathrm{D}_{\text {mean }}$ of the spinal cord $(\mathrm{p}<0.05)$. These results showed a strong correlation (i.e., $r>0.7$ ) according to the magnitude determined by Infusino et al., whereas other structures corresponded to weak and moderate correlation. On the other hand, percentage dose differences in the cases of prostate cancer were larger than those of HN cases. There was no statistical significance, except for the maximum dose difference of the bladder. Weak and moderate correlation was shown for all target and structure volumes of in the case of prostate cancer, in accordance with the previous study. Figures 3 and 4 show the relation between percentage dose difference and the gamma pass rate for each volume. It can be seen that the percentage dose difference for the mean dose of PTV decreases with the increasing the gamma pass rate. However, there was no apparent correlation for other volumes.

\section{Discussion}

Patient-specific QA is essential for confirming the machine status and accuracy of dose delivery before radiation treatment, and it has been accomplished with various devices such as the ionizing chamber, film, and 2D array detectors. In this study, pretreatment QA was performed with three dosimetric devices, and analyzed using each softwares. Moreover, percentage dose difference and correlation with the mean gamma pass rates were evaluated for target and organ volumes by $3 \mathrm{DVH}$ software. To this end, the portal dosimetry system that uses EPID provides a very simple approach, as well as and it has high resolution.

Sharma et al. evaluated the efficiency of portal dosimetry using the gamma index, and compared these results with the MatriXX 2D ion chamber array. They reported that the portal dosimetry can be used as an alternative process $[17,18]$. The portal dosimetry system provided by VARIAN was also compared with EPIDose and MapCHECK (Sun Nuclear Corporation, Melbourne, FL, USA) according to dosimetric characteristics [19]. They reported that the PD algorithm shows similar results to those of EPIDose and MapCHECK for absolute dose gamma evaluation. However, it shows different results for items of off-axis field in comparison with EPIDose and MapCHECK. It is necessary to demonstrate for various items of IMRT QA. The MatriXX 2D ion chamber array was compared with the film, PTW Seven29 array and the Delta4 array for RapidArc and IMRT plan [20]. In the study, they determined that all commercial devices can be used for routine patientspecific QA.

On the other hand, the ArcCHECK 2D diode array is a detector array specifically designed for VMAT treatment. Li et al. evaluated for dosimetric characteristics of the IMRT and VMAT verification plans [21]. Their study reported that the ArcCHECK detector suitably functions for plan verification. The dose distribution measured with the ArcCHECK array was investigated for VMAT plan on 3DVH software [22]. They evaluated the correlation between the percentage dose difference and gamma pass rate for cases of nasopharyngeal cancer and esophageal cancer. They concluded that a high dose difference error was observed for some DVH-based metrics. However, 3DVH software still provides useful information. Furthermore, the Compass system (IBA Dosimetry, GmbH, Germany) is able to reconstruct the 3D dose distribution such as $3 \mathrm{DVH}$ software. This system offers accurate dose calculation for phantom studies as well as IMRT QA [23].

The purpose of this study is to confirm the IMRT QA with various $2 \mathrm{D}$ array detectors, and to compare the results with those of

\begin{tabular}{|c|c|c|c|c|c|c|c|}
\hline \multicolumn{1}{|c|}{} & $3 \mathrm{~mm} / 3 \%$ & $\mathrm{SD}$ & $2 \mathrm{~mm} / 2 \%$ & $\mathrm{SD}$ & $1 \mathrm{~mm} / 1 \%$ & SD \\
\hline \multirow{4}{*}{$\mathrm{HN}$} & MatriXX & 98.51 & 0.96 & 93.44 & 2.80 & 70.63 & 5.24 \\
\cline { 2 - 8 } & PD & 99.56 & 0.34 & 97.11 & 1.74 & 80.11 & 5.13 \\
\cline { 2 - 8 } & ArcCHECK & 98.35 & 1.13 & 84.64 & 8.01 & 42.01 & 9.07 \\
\cline { 2 - 8 } & 3DVH(2D) & 97.97 & 1.76 & 82.37 & 9.70 & 36.90 & 6.80 \\
\cline { 2 - 8 } & 3DVH(3D) & 98.79 & 2.38 & 94.80 & 8.05 & 77.56 & 17.90 \\
\hline \multirow{5}{*}{ Prostate } & MatriXX & 98.00 & 1.89 & 91.74 & 3.90 & 60.14 & 6.15 \\
\cline { 2 - 8 } & PD & 97.72 & 0.75 & 89.01 & 2.36 & 59.27 & 5.65 \\
\cline { 2 - 8 } & ArcCHECK & 97.85 & 1.41 & 89.57 & 3.97 & 53.11 & 4.22 \\
\cline { 2 - 8 } & 3DVH(2D) & 96.46 & 2.07 & 86.06 & 4.74 & 50.14 & 3.24 \\
\cline { 2 - 8 } & 3DVH(3D) & 94.50 & 0.72 & 86.66 & 1.95 & 53.00 & 4.24 \\
\hline
\end{tabular}

Table 1: Gamma pass rates and standard deviations. HN: Head and Neck; SD Standard Deviation. 
Citation: Park JH, Kim TG, Min SK, Park BD (2016) Comparison of 3DVH Software with Two-dimensional Array Systems on Pretreatment Verification for Volumetric-modulated Arc Therapy. J Nucl Med Radiat Ther 7: 284. doi:10.4172/2155-9619.1000284

Page 4 of 6

\begin{tabular}{|c|c|c|c|c|c|c|c|c|c|}
\hline \multicolumn{5}{|c|}{$\mathrm{HN}$} & \multicolumn{5}{|c|}{ Prostate } \\
\hline Structure & Parameter & $\% D D$ & $r$ & $\mathrm{p}$-value & Structure & Parameter & $\% D D$ & $r$ & $p$-value \\
\hline \multirow{5}{*}{ PTV } & Dmean & 1.20 & 0.915 & 0.001 & \multirow{5}{*}{ PTV } & Dmean & 4.47 & 0.606 & 0.063 \\
\hline & Dmax & 0.52 & 0.708 & 0.033 & & Dmax & 12.58 & 0.419 & 0.228 \\
\hline & $D 2 \%$ & 0.79 & 0.971 & 0.000 & & $D 2 \%$ & 8.80 & 0.471 & 0.170 \\
\hline & D95\% & 1.55 & 0.830 & 0.006 & & D95\% & 2.76 & 0.532 & 0.114 \\
\hline & $D 98 \%$ & 1.54 & 0.797 & 0.010 & & $D 98 \%$ & 3.04 & 0.576 & 0.082 \\
\hline \multirow{2}{*}{ Spinal cord } & Dmean & 0.76 & 0.911 & 0.001 & \multirow{2}{*}{ Penile bulb } & Dmean & 6.17 & 0.140 & 0.700 \\
\hline & $D \max$ & 0.98 & 0.481 & 0.190 & & $D \max$ & 12.08 & 0.036 & 0.922 \\
\hline \multirow{2}{*}{ Rt parotid } & Dmean & 1.04 & 0.052 & 0.895 & \multirow{2}{*}{ Bladder } & Dmean & 5.71 & 0.100 & 0.783 \\
\hline & $D \max$ & 2.48 & 0.544 & 0.130 & & $D \max$ & 11.32 & 0.688 & 0.028 \\
\hline \multirow{6}{*}{ Lt parotid } & Dmean & 3.99 & 0.190 & 0.624 & \multirow{2}{*}{ Rt femur } & Dmean & 3.37 & 0.603 & 0.065 \\
\hline & Dmax & 2.18 & 0.171 & 0.660 & & $D \max$ & 5.30 & 0.229 & 0.525 \\
\hline & & & & & \multirow{2}{*}{ Lt femur } & Dmean & 3.18 & 0.145 & 0.690 \\
\hline & & & & & & Dmax & 5.12 & 0.034 & 0.926 \\
\hline & & & & & \multirow{2}{*}{ Rectum } & Dmean & 5.63 & 0.027 & 0.940 \\
\hline & & & & & & $D \max$ & 8.20 & 0.030 & 0.935 \\
\hline
\end{tabular}

Table 2. Percentage dose difference and correlation between \%DD and gamma pass rate. $r=$ Pearson correlation coefficient.
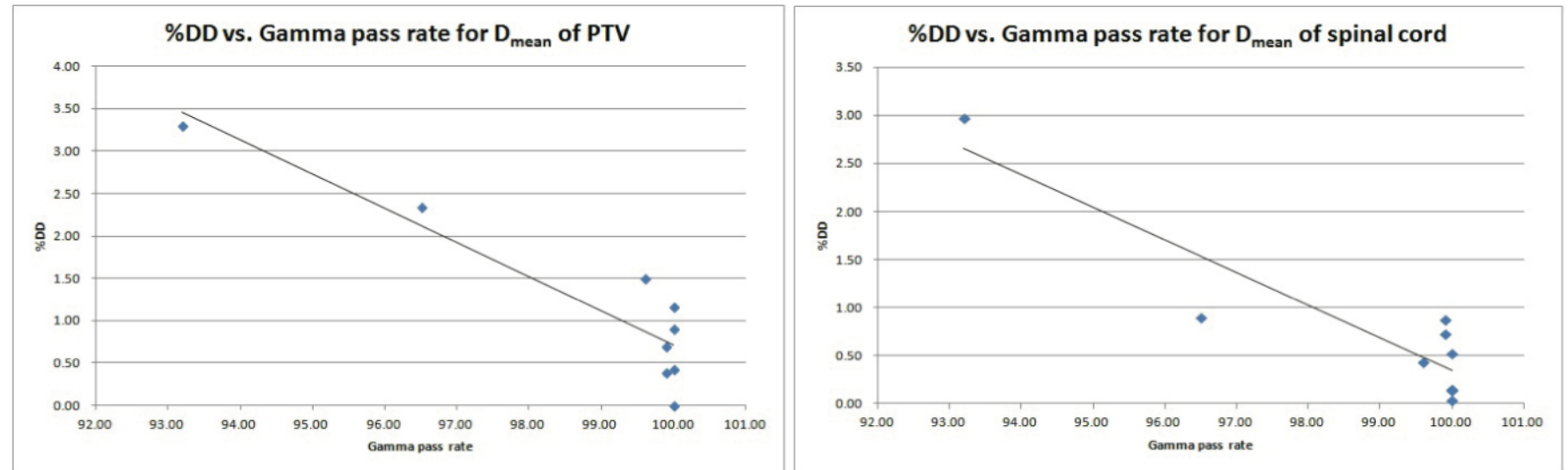

\%DD vs. Gamma pass rate for $D_{\text {mean }}$ of right parotid
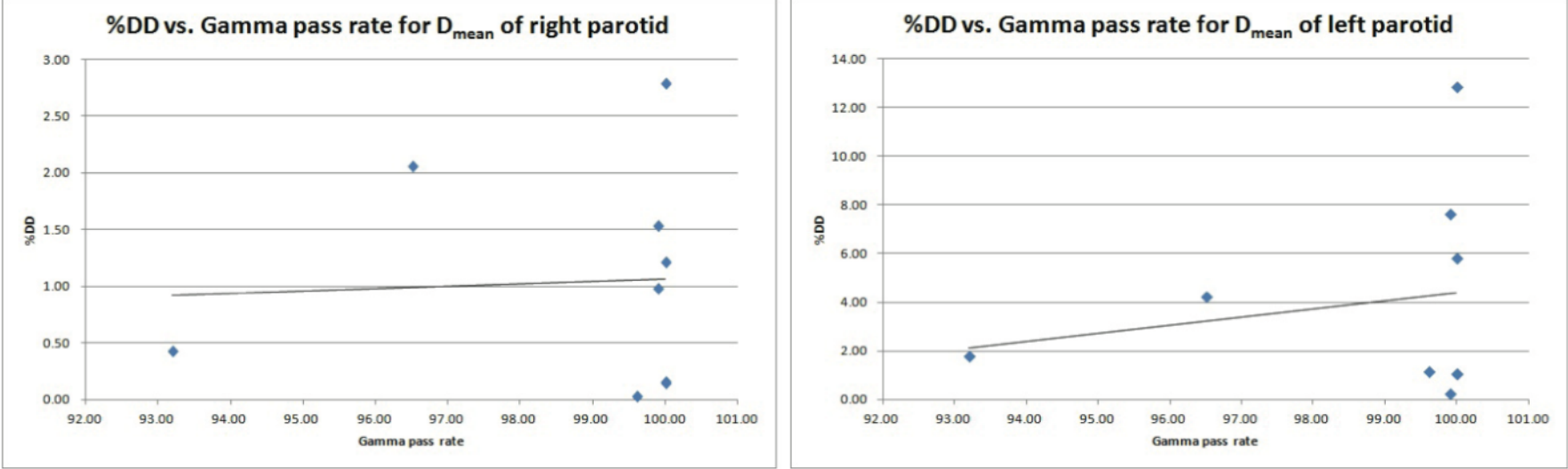

Figure 3: Correlation between percentage dose difference and gamma pass rate for each structure in the cases of head and neck (HN) cancer.

the $3 \mathrm{DVH}$ software. The gamma passing rate evaluated with $2 \mathrm{D}$ array detectors corresponded to more than $95 \%$ for the $3 \% / 3 \mathrm{~mm}$ criterion. Although the results for the cases of $\mathrm{HN}$ cancer matched well, large dose differences were observed in the case of prostate cancer. So we think that we should investigate the correlation between the \%DD and gamma pass rate in further studies.

\section{Conclusion}

In this study, we evaluated various dosimetry systems for VMAT verification plans. The dosimetry systems yielded similar results for the gamma pass rate using the $3 \% / 3 \mathrm{~mm}$ criterion. Using $3 \mathrm{DVH}$ software, we were able to estimate the accuracy of dose distribution through the DVH for target and normal organ volumes. The mean gamma pass rates exceeded $95 \%$ for the $3 \% / 3 \mathrm{~mm}$ criterion, except for the value of $3 \mathrm{DVH}(3 \mathrm{D})$. In the cases of $\mathrm{HN}$ cancer, the mean gamma pass rates of ArcCHECK and 3DVH (2D) were estimated to be lower than 
Citation: Park JH, Kim TG, Min SK, Park BD (2016) Comparison of 3DVH Software with Two-dimensional Array Systems on Pretreatment Verification for Volumetric-modulated Arc Therapy. J Nucl Med Radiat Ther 7: 284. doi:10.4172/2155-9619.1000284
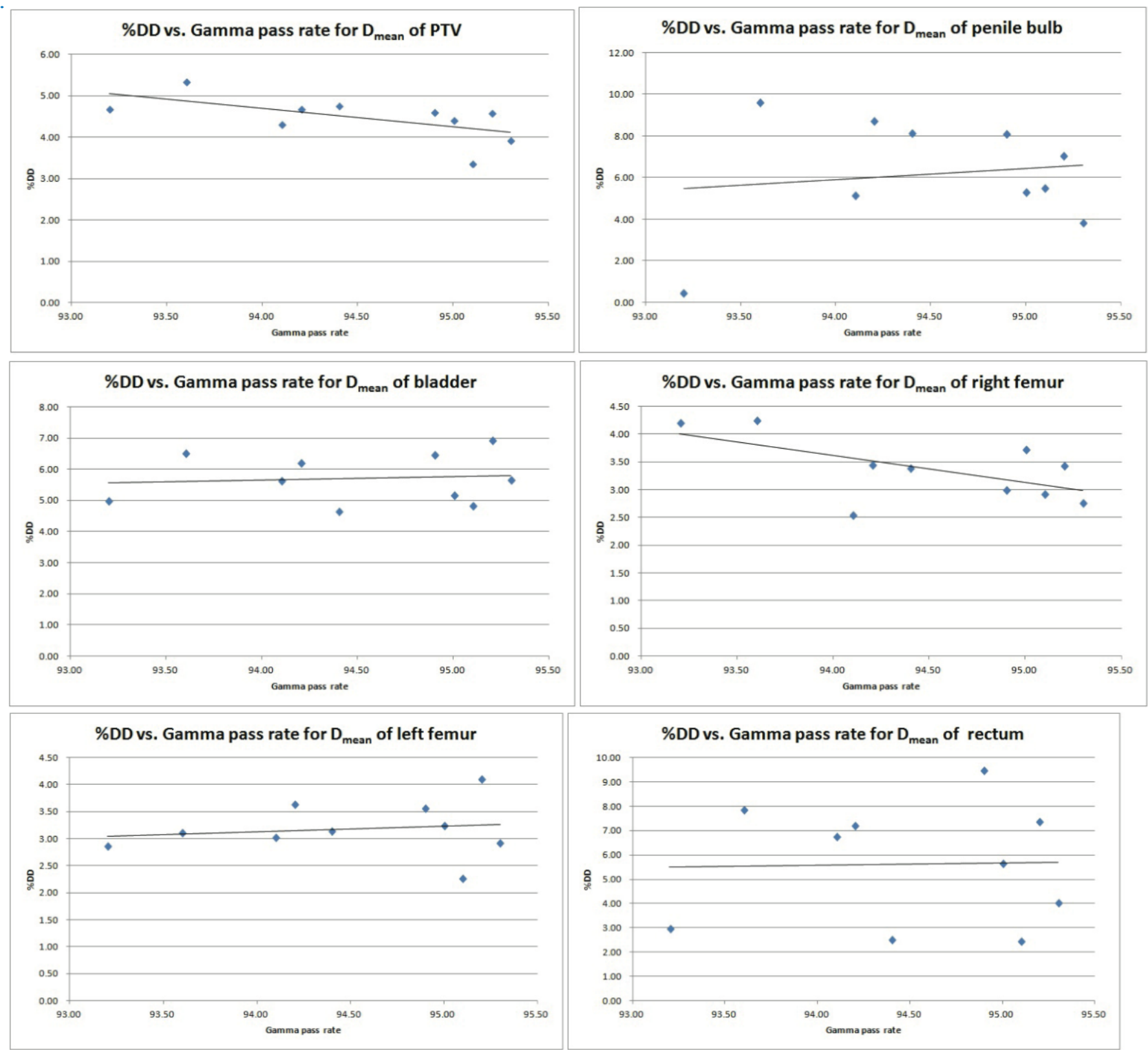

Figure 4: Correlation between percentage dose difference and gamma pass rate for each structure in the cases of prostate cancer

those of MatriXX, PD, and 3DVH (3D) with $2 \% / 2 \mathrm{~mm}$ and $1 \% / 1 \mathrm{~mm}$ criteria. The mean gamma values presented similar results with each criterion for the cases of prostate cancer. In $\mathrm{HN}$ cases, the percentage dose difference was less than $4 \%$ for all structures, and the percentage dose difference was correlated with the gamma pass rate for PTV and $\mathrm{D}_{\text {mean }}$ of the spinal cord $(\mathrm{p}<0.05)$. The percentage dose difference was larger for cases of prostate cancer than for cases of HN cancer. Furthermore, there was no statistical significance found, except for the $\mathrm{D}_{\max }$ of the bladder. Additionally, we found large differences for the cases of prostate cancer by comparing the DVH results using $3 \mathrm{DVH}$. Judging from our results, the use of $3 \mathrm{DVH}$ software requires further investigation and discussion.

\section{Acknowledgements}

We would like to express our deepest gratitude to Dr. Jung-Won Kwak for his comments on the manuscript.

\section{References}

1. Langer M, Leong J (1987) Optimization of beam weights under dose-volume restrictions. Int J Radiat Oncol Biol Phys 13: 1255-1260.

2. Mok H, Crane $\mathrm{CH}$, Palmer MB, Briere TM, Beddar S, et al. (2011) Intensity modulated radiation therapy (IMRT): differences in target volumes and improvement in clinically relevant doses to small bowel in rectal carcinoma. Radiat Oncol 6: 63

3. Mundt AJ, Lujan AE, Rotmensch J, Waggoner SE, Yamada SD, et al. (2002) Intensity-modulated whole pelvic radiotherapy in women with gynecologic malignancies. Int J Radiat Oncol Biol Phys 52: 1330-1337.

4. Chen YJ, Liu A, Tsai PT, Vora NL, Pezner RD, et al. (2005) Organ sparing by conformal avoidance intensity-modulated radiation therapy for anal cancer: dosimetric evaluation of coverage of pelvis and inguinal/femoral nodes. Int $J$ 
Citation: Park JH, Kim TG, Min SK, Park BD (2016) Comparison of 3DVH Software with Two-dimensional Array Systems on Pretreatment Verification for Volumetric-modulated Arc Therapy. J Nucl Med Radiat Ther 7: 284. doi:10.4172/2155-9619.1000284

Radiat Oncol Biol Phys 63: 274-281

5. Menkarios C, Azria D, Laliberte B, Moscardo CL, Gourgou S, et al. (2007) Optimal organ-sparing intensity-modulated radiation therapy (IMRT) regimen for the treatment of locally advanced anal canal carcinoma: a comparison of conventional and IMRT plans. Radiat Oncol 2: 41.

6. Otto K (2008) Volumetric modulated arc therapy: IMRT in a single gantry arc. Med Phys 35: 310-317.

7. (2010) Prescribing, Recording, and Reporting Photon-Beam IntensityModulated Radiation Therapy (IMRT): Contents. J ICRU 10: NP.

8. Ezzell GA, Burmeister JW, Dogan N, LoSasso TJ, Mechalakos JG, et al. (2009) IMRT commissioning: multiple institution planning and dosimetry comparisons, a report from AAPM Task Group 119. Med Phys 36: 5359-5373.

9. Fenoglietto P, Laliberte B, Ailleres N, Riou O, Dubois JB, et al. (2011) Eight years of IMRT quality assurance with ionization chambers and film dosimetry: experience of the Montpellier Comprehensive Cancer Center. Radiat Oncol 6: 85.

10. Zhen $\mathrm{H}$, Nelms BE, Tome WA (2011) Moving from gamma passing rates to patient DVH-based QA metrics in pretreatment dose QA. Med Phys 38: 5477-5489.

11. Keeling VP, Ahmad S, Algan O, Jin H (2014) Dependency of planned dose perturbation (PDP) on the spatial resolution of MapCHECK 2 detectors. J Appl Clin Med Phys 15: 4457.

12. Infusino E, Mameli A, Conti R, Gaudino D, Stimato G, et al. (2014) Initial experience of ArcCHECK and 3DVH software for RapidArc treatment plan verification. Med Dosim 39: 276-281.

13. Olch AJ (2012) Evaluation of the accuracy of 3DVH software estimates of dose to virtual ion chamber and film in composite IMRT QA. Med Phys 39: 81-86.

14. Song JH, Shin HJ, Kay CS, Son SH (2015) Dosimetric verification by using the ArcCHECK system and 3DVH software for various target sizes. PLoS One 10: e0119937.
15. Nelms BE, Simon WE (2011) Radiation Therapy Plan Dose Perturbation System Method. United States Ptent US 7945022: 1-15.

16. Both S, Alecu IM, Stan AR, Alecu M, Ciura A, et al. (2007) A study to establish reasonable action limits for patient-specific quality assurance in intensitymodulated radiation therapy. J Appl Clin Med Phys 8: 1-8.

17. Kim YL, Chung JB, Kim JS, Lee JW, Choi KS (2014) Comparison of the performance between portal dosimetry and a commercial two-dimensional array system on pretreatment quality assurance for volumetric-modulated arc and intensity-modulated radiation therapy. J Korean Physical Soc 64: 12071212.

18. Sharma DS, Mhatre V, Heigrujam M, Talapatra K, Mallik S (2010) Portal dosimetry for pretreatment verification of IMRT plan: a comparison with 2D ion chamber array. J Appl Clin Med Phys 11: 3268.

19. Bailey DW, Kumaraswamy L, Bakhtiari M, Malhotra HK, Podgorsak MB (2012) EPID dosimetry for pretreatment quality assurance with two commercial systems. J Appl Clin Med Phys 13: 3736.

20. Chandraraj V, Stathakis S, Manickam R, Esquivel C, Supe SS, et al. (2011) Comparison of four commercial devices for RapidArc and sliding window IMRT QA. J Appl Clin Med Phys 12: 3367.

21. Li G, Zhang Y, Jiang X, Bai S, Peng G, et al. (2013) Evaluation of the ArcCHECK QA system for IMRT and VMAT verification. Phys Med 29: 295-303.

22. Jin X, Yan H, Han C, Zhou Y, Yi J, et al. (2015) Correlation between gamma index passing rate and clinical dosimetric difference for pre-treatment $2 \mathrm{D}$ and $3 \mathrm{D}$ volumetric modulated arc therapy dosimetric verification. $\mathrm{Br} \mathrm{J}$ Radiol 88 20140577

23. Nakaguchi $Y$, Ono T, Maruyama M, Nagasue N, Shimohigashi $Y$, et al. (2015) Validation of fluence-based 3D IMRT dose reconstruction on a heterogeneous anthropomorphic phantom using Monte Carlo simulation. J Appl Clin Med Phys 16: 5199. 